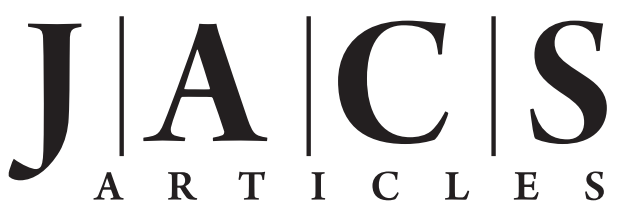

Published on Web 09/23/2010

\title{
Weave Tile Architecture Construction Strategy for DNA Nanotechnology
}

\author{
Majken N. Hansen, ${ }^{\dagger}$ Alex M. Zhang, ${ }^{\ddagger}$ Abhijit Rangnekar, ${ }^{\dagger, \|}$ Kristin M. Bompiani, ${ }^{\S}$ \\ Joshua D. Carter," Kurt V. Gothelf, ${ }^{\dagger}$ and Thomas H. LaBean ${ }^{\star, \|}$ \\ Centre for DNA Nanotechnology, Department of Chemistry and iNANO, Aarhus University, \\ 8000 Arhus C, Denmark, University Program in Genetics and Genomics and Department of \\ Surgery, Duke University Medical Center, Trinity College, Duke University, and Departments of \\ Computer Science, Chemistry, and Biomedical Engineering, Duke University,
} Durham, North Carolina 27708

Received June 2, 2010; E-mail: thomas.labean@ duke.edu

\begin{abstract}
Architectural designs for DNA nanostructures typically fall within one of two broad categories: tile-based designs (assembled from chemically synthesized oligonucleotides) and origami designs (woven structures employing a biological scaffold strand and synthetic staple strands). Both previous designs typically contain many Holliday-type multi-arm junctions. Here we describe the design, implementation, and testing of a unique architectural strategy incorporating some aspects of each of the two previous design categories but without multi-arm junction motifs. Goals for the new design were to use only chemically synthesized DNA, to minimize the number of component strands, and to mimic the back-and-forth, woven strand routing of the origami architectures. The resulting architectural strategy employs "weave tiles" formed from only two oligonucleotides as basic building blocks, thus decreasing the burden of matching multiple strand stoichiometries compared to previous tile-based architectures and resulting in a structurally flexible tile. As an example application, we have shown that the four-helix weave tile can be used to increase the anticoagulant activity of thrombin-binding aptamers in vitro.
\end{abstract}

\section{Introduction}

The use of DNA as a construction material for the design of nanostructures and the assembly of particles and biomolecules has recently attracted much attention. ${ }^{1}$ Due to the predictable and programmable Watson-Crick base paring, stability, high persistence length of the double helix (dsDNA), and ease of synthesis of oligonucleotides (up to $\sim 200$ bases long), DNA nanotechnology has become a major direction for self-assemblybased (bottom up) fabrication in bionanotechnology.

Architectural designs for DNA nanostructures typically fall within two broad categories: tile-based designs and origami designs, both of which typically contain many Holliday-type multi-arm junctions (where two strands participate in reciprocal exchange between dsDNA domains). More recently, halfcrossovers (where only a single strand crosses over between dsDNA domains) have also come into play. $^{2}$

In tile-based approaches, two or more DNA double helices are held together by branch junction crossovers, creating stiff

\footnotetext{
$\pitchfork$ Aarhus University.

$\$$ Duke University Medical Center.

$\doteqdot$ Trinity College, Duke University.

"Departments of Computer Science, Chemistry, and Biomedical Engineering, Duke University.

(1) (a) Seeman, A. C. Nature 2003, 421, 427-431. (b) Gothelf, K. V.; LaBean, T. H. Org. Biomol. Chem. 2005, 3, 4023-4037. (c) Li, H. Y.; Carter, J. D.; LaBean, T. H. Mater. Today 2009, 12, 20-28. (d) Amin, R.; Kim, S.; Park, S. H.; LaBean, T. H. NANO 2009, 4, 119-139.

(2) Yin, P.; Hariadi, R. F.; Sahu, S.; Choi, H. M. T.; Park, S. H.; LaBean, T. H.; Reif, J. H. Science 2008, 321, 824-826.
}

tiles with high thermal stability. ${ }^{3}$ Using single-stranded overhangs (sticky-ends), these simple tiles can form large one- and two-dimensional DNA arrays. ${ }^{3}$ Other tile varieties, such as the six-helix bundle, ${ }^{4}$ the $4 \times 4$ cross tile, ${ }^{5}$ the tensegrity triangle, ${ }^{6}$ and the three-layered logpile, ${ }^{7}$ have also been demonstrated, and by application of DNA sequence symmetry, tiles consisting of only two ${ }^{8}$ or three ${ }^{9}$ strands have been created. Very recently, the first tile-based 3D crystals have also been reported. ${ }^{10}$

Origami strategies employ a biological scaffold strand (e.g., M13 bacteriophage single-strand genome) held together by multiple synthetic staple strands in a woven fashion, creating sheets that are roughly $100 \mathrm{~nm}$ in diameter, with a spatial

(3) (a) Li, X.; Yang, X.; Qi, J.; Seeman, N. C. J. Am. Chem. Soc. 1996, 118, 6131-6140. (b) Winfree, E.; Liu, F.; Wenzler, L. A.; Seeman, N. C. Nature 1998, 394, 539-544. (c) Mao, C.; Sun, W.; Seeman, N. C. J. Am. Chem. Soc. 1999, 121, 5437-5443. (d) LaBean, T. H.; Yan, H.; Kopatsch, J.; Liu, F.; Winfree, E.; Reif, J. H.; Seeman, N. C. J. Am. Chem. Soc. 2000, 122, 1848-1860.

(4) Mathieu, F.; Liao, S.; Mao, C.; Kopatsch, J.; Wang, T.; Seeman, N. C. Nano Lett. 2005, 5, 661-665.

(5) Yan, H.; Park, S. H.; Finkelstein, G.; Reif, J. H.; LaBean, T. H. Science 2003, 301, 1882-1884.

(6) Liu, D.; Wang, W.; Deng, Z.; Walulu, R.; Mao, C. J. Am. Chem. Soc. 2004, 126, 2324-2325.

(7) Malo, J.; Mitchell, J. C.; Tuberfield, A. J. J. Am. Chem. Soc. 2009, $131,13574-13575$.

(8) Liu, H.; Ribbe, A. E.; Mao, C. Biomacromolecules 2005, 6, $2943-$ 2945.

(9) He, Y.; Tian, Y.; Chen, Y.; Deng, Z.; Ribbe, A. E.; Mao, C. Angew. Chem., Int. Ed. 2005, 44, 6694-6696.

(10) Zheng, J.; Birktoft, J. J.; Chen, Y.; Wang, T.; Sha, R.; Constantinou, P. E.; Ginell, S. L.; Mao, C.; Seeman, N. C. Nature 2009, 461, 7477. 
resolution of about $6 \mathrm{~nm} .{ }^{11}$ This strategy has also recently been applied to create $3 \mathrm{D}$ structures. ${ }^{12,13}$

Both design types have been used to organize biomolecules and inorganic nanoparticles. The most explored method utilizes covalent modification of DNA strands with biotin moieties and subsequent binding of streptavidin to the assembled structures in any pattern desired. ${ }^{5,14}$ Aptamers, which are short DNA or RNA sequences that can bind other nucleic acids, proteins, small organic compounds, and even entire organisms, ${ }^{15,16}$ can be incorporated into the DNA sequences and have been used to bind thrombin and antibodies to the DNA scaffolds. ${ }^{17}$ Gold particles conjugated to thiol-modified DNA have been organized on DNA lattices by annealing to single-stranded overhangs in the arrays. ${ }^{18}$

Here we describe the design, implementation, and testing of a unique architectural strategy incorporating some aspects of each of the two previous design categories but with flexible tethering between helices and completely devoid of Holliday junction-like crossovers. Initial goals for the new design were to use only chemically synthesized DNA, to minimize the number of component strands, and to mimic the back-and-forth, woven strand routing of the origami architectures. The resulting architectural strategy employs "weave tiles" and utilizes basic building blocks (tiles) formed from only two oligonucleotides. We have demonstrated formation of several tile types and their assembly into extended lattices of moderate sizes. We have further shown that the four-helix weave tile (4HT) can be used as a nanoscale platform to organize thrombin-binding aptamers and increase their anticoagulant activity in vitro.

\section{Materials and Methods}

DNA Strands. Synthetic oligonucleotides (sequences given in Table 1) were purchased from Integrated DNA Technologies (Coralville, IA) and purified by polyacrylamide gel electrophoresis (PAGE). Tiles and lattices were formed by annealing a stoichiometric quantity of the two strands in physiological buffer, 1xTAE/ $\mathrm{Mg}^{2+}$ (40 mM Tris acetate ( $\left.\mathrm{pH} 8.0\right), 2 \mathrm{mM}$ EDTA, and $12.5 \mathrm{mM}$ magnesium acetate), at a concentration of $1 \mu \mathrm{M}$. For the annealing, the mixed strand solution was cooled slowly from 95 to $35^{\circ} \mathrm{C}$ by placing the sealed microcentrifuge tube in $1 \mathrm{~L}$ of boiled water in a closed Styrofoam box overnight, followed by incubation at $4{ }^{\circ} \mathrm{C}$ for at least $6 \mathrm{~h}$ before atomic force microscopy (AFM) imaging.

AFM Imaging. AFM imaging was performed in tapping mode under 1xTAE/ $\mathrm{Mg}^{2+}$ buffer. One microliter of the annealed sample was diluted with $4 \mu \mathrm{L}$ of buffer and placed on freshly cleaved mica

(11) Rothemund, P. W. K. Nature 2006, 440, 297-302.

(12) Douglas, S. M.; Dietz, H.; Liedl, T.; Högberg, B.; Graf, F.; Shih, W. M. Nature 2009, 459, 414-418.

(13) Andersen, E. S.; Dong, M.; Nielsen, M. M.; Jahn, K.; Subramani, R.; Mamdouh, W.; Golas, M. M.; Sander, B.; Stark, H.; Oliveira, C. L. P.; Pedersen, J. S.; Birkedal, V.; Besenbacher, F.; Gothelf, K. V.; Kjems, J. Nature 2009, 459, 73-77.

(14) Park, S. H.; Yin, P.; Liu, Y.; Reif, J. H.; LaBean, T. H.; Yan, H. Nano Lett. 2005, 5, 729-733.

(15) Brody, E. N.; Willis, M. C.; Smith, J. D.; Jayasena, S.; Zichi, D.; Gold, L. Mol. Diagn. 1999, 4, 336-388.

(16) Conrad, R. C.; Giver, L.; Tian, Y.; Ellington, A. D. Comb. Chem. 1996, 267, 336-367.

(17) (a) Liu, Y.; Lin, C.; Li, H.; Yan, H. Angew. Chem., Int. Ed. 2005, 44, 4333-4338. (b) Lin, C.; Katilius, E.; Zhang, J.; Yan, H. Angew. Chem. Int. Ed. 2006, 45, 5296-5301. (c) Li, H.; LaBean, T. H.; Kenan, D. Org. Biomol. Chem. 2006, 4, 3420-3426. (d) Rinker, S.; Ke, Y.; Liu, Y.; Chhabra, R.; Yan, H. Nature Nanotechnol. 2008, 3, 418-422.

(18) (a) Le, J. D.; Pinto, Y.; Seeman, N. C.; Musier-Forsyth, K.; Taton, T. A.; Kiehl, R. A. Nano Lett. 2004, 4, 2343. (b) Zhang, J. P.; Liu, Y.; Ke, Y. G.; Yan, H. Nano Lett. 2006, 6, 248. (c) Sharma, J.; Chhabra, R.; Andersen, C. S.; Gothelf, K. V.; Yan, H.; Liu, Y. J. Am Chem. Soc. 2008, 130, 7820-7821. for $5 \mathrm{~min}$, and then $30 \mu \mathrm{L}$ of $1 \times \mathrm{TAE} / \mathrm{Mg}^{2+}$ was placed on the mica and another $30 \mu \mathrm{L}$ on the AFM tip. AFM images were obtained on a Digital Instruments Nanoscope IIIa (Veeco) with a multimode head and fluid cell using NP-S oxide-sharpened silicon nitride tips (Vecco).

Thermal Melts. The melting profiles of the DNA tile and lattice were measured at $260 \mathrm{~nm}$ on a Cary 100Bio UV-vis spectrophotometer utilizing a quartz cell with $1 \mathrm{~cm}$ path length. The concentration of each DNA strand was $1 \mu \mathrm{M}$ and the heating rate $0.5^{\circ} \mathrm{C} / \mathrm{min}$.

Coagulation Assay. A model ST4 coagulometer (Diagnostica Stago) was used to run an activated partial thromboplastin-time (aPTT) assay to test the clotting time for each sample. The reagents used include normal pooled plasma from George King Bio-Medical, as well as a $\mathrm{CaCl}_{2}$ solution and TriniClot aPTT reagent from Trinity Biotech. Samples were activated by the TriniClot aPTT reagent, and clotting was initiated by addition of the $\mathrm{CaCl}_{2}$ solution. Initially, the plasma was thawed at $37^{\circ} \mathrm{C}$ and then placed in ice. The platelin and $\mathrm{CaCl}_{2}$ solutions were then drawn into separate automated pipettors with the $\mathrm{CaCl}_{2}$ solution kept in a heated slot at $37{ }^{\circ} \mathrm{C}$. Cuvettes were placed in designated slots, with one metal bead placed in each well. The next step involved pipetting $50 \mu \mathrm{L}$ of plasma into each well, adding $50 \mu \mathrm{L}$ of the TriniClot reagent, and then rocking the cuvette to mix the solution. The mixture was then incubated for $5 \mathrm{~min}$ at $37^{\circ} \mathrm{C}$ before the addition of $5 \mu \mathrm{L}$ of either the aptamer dilution or buffer for the negative control baseline. The sample was once again mixed by rocking and then incubated for an additional $5 \mathrm{~min}$ to allow aptamer/protein interactions. To initiate the clotting assay, $50 \mu \mathrm{L}$ of $\mathrm{CaCl}_{2}$ was added to each well. The machine then began to measure the clotting time on the basis of the movement of the metal bead in each well. Once clotting had occurred, the bead was immobilized and the time was recorded. Each test was conducted in triplicate to ensure reliable results.

\section{Results and Discussion}

Recombining sequences from a previously published DDX tile, ${ }^{19}$ two new tile designs comprised of four or eight helices were made. Each helical layer had a length of 1.5 helical turns (16 bases) and was linked with short T-loop sequences to create bulges in the helix ends, thus connecting adjacent helices while simultaneously eliminating helix stacking interactions. Helix stacking between neighboring nanostructures is commonly observed with origami designs and tile-based lattices with straight sides and unprotected, hydrophobic helix ends. ${ }^{11,20}$ Also, 10-base sticky-ends of a repeated AT sequence were added at each tile corner. The repeated sticky-ends were chosen for two reasons. First, they made the spatial orientation of each tile less relevant, since hybridization with a neighboring tile was possible regardless of rotational orientation of the tiles. Second, they made it possible for the base pairs to "slide" register, thus making the initiation of the annealing easier and adding flexibility of length within tile association points. Since only a slight interaction of a few base pairs of the sticky-ends might hold the tiles loosely together, the system gains some addition time for the formation of the full and more thermodynamically favorable hybridization.

The tiles were annealed by heating to $90{ }^{\circ} \mathrm{C}$ and slow cooling in a styrofoam box over $24 \mathrm{~h}$. PAGE gels (Figure 1) for the four-helix tile (4HT) showed that the individual tiles did form. Side products where the two "flanking" helixes annealed first, thereby not allowing the central helixes to form, were not observed, as the gels only showed one distinct band. On the

(19) Reishus, D.; Shaw, B.; Brun, Y.; Chelyapov, N.; Adleman, L. J. Am. Chem. Soc. 2005, 127, 17590-17591.

(20) Yan, H.; LaBean, T. H.; Feng, L.; Reif, J. H. Proc. Natl. Acad. Sci. U.S.A. 2003, 100, 8103-8108. 


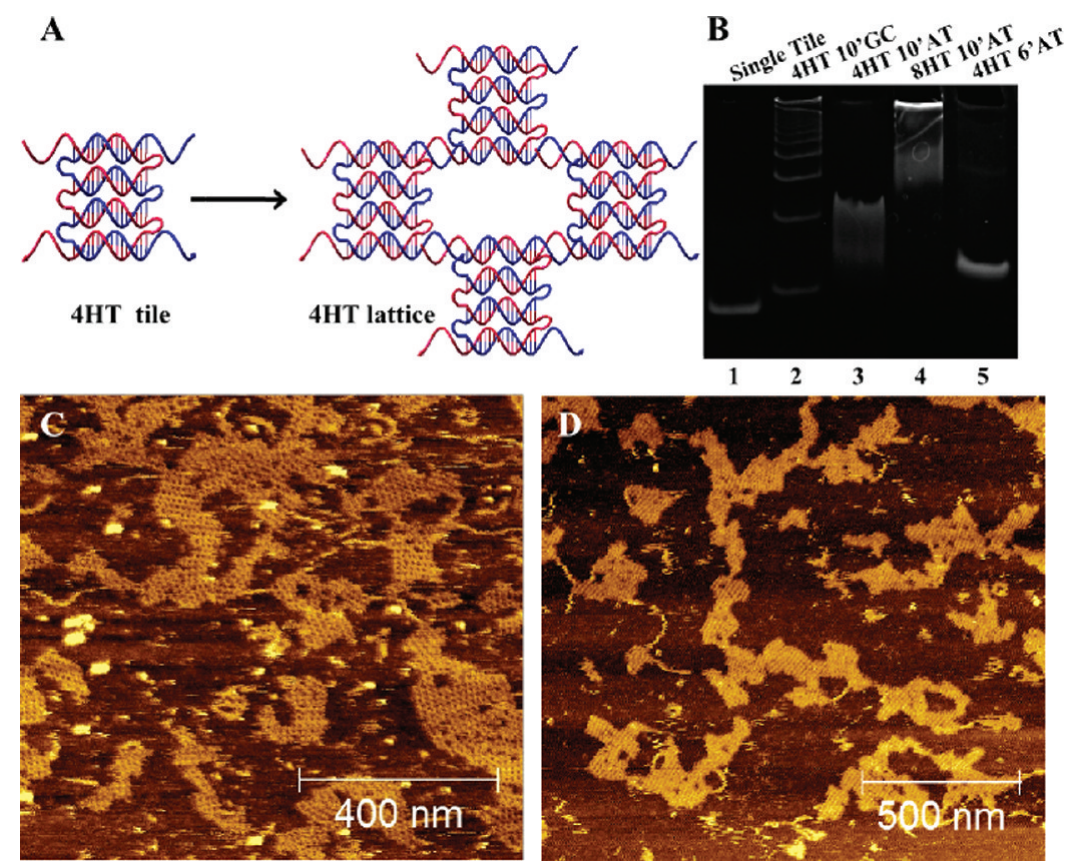

Figure 1. (A) Schematic drawings of four-helix weave tile (4HT) and the designed assembly of four such tiles into a small fragment of lattice. (B) $7 \%$ PAGE gel showing well-formed single 4HT tiles without sticky-ends (lane 1), a range of sizes of 4HT lattices formed from tiles with 10 bp GC sticky-ends (lane 2), 4HT lattices with 10 bp AT sticky-ends (lane 3), 8HT arrays with 10 bp AT sticky-ends (lane 4), and 4HT arrays with 6 bp AT sticky-ends (lane 5). (C,D) Typical AFM images of 4HT lattice.
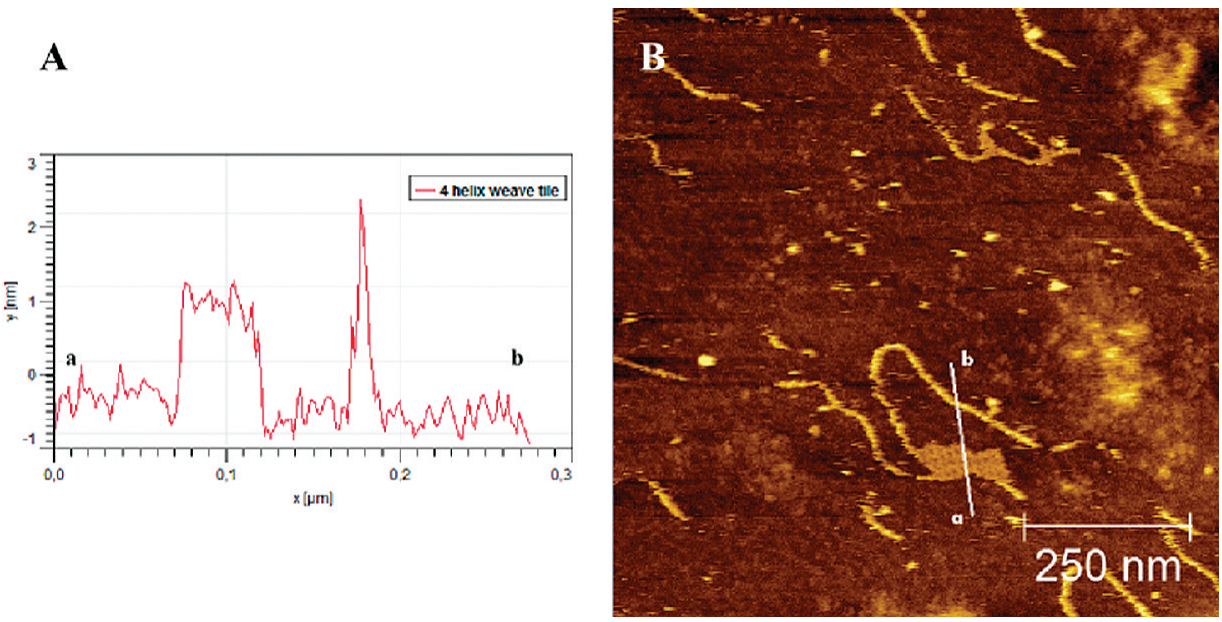

Figure 2. (A) Height profile of the lattice and ribbons following the line presented in panel B. The ribbons had a height of approximately $2.2 \mathrm{~nm}$, which was about twice as high as for the tile array $(1.2 \mathrm{~nm}$ ), suggesting a double-layered twisted formation. (B) AFM image (collected under buffer) of small lattice and tube-like structures formed from annealed four-helix weave tiles.

the 2D array has a lower conformational free energy. No array structures were observed when the sticky-ends were shortened to five or six bases. In an attempt to raise the stability of the array, sticky-ends were changed to GC sequences. In the gel electrophoresis analysis (Figure 1B), this gave a distinct ladder and an expectation of larger array structures, but only inferior lattice formation was observed by AFM. Further investigations involving other sticky-end lengths and non-repetitive sequences will be followed up on but are beyond the scope of the present report.

(21) (a) Mitchell, J. C.; Harris, J. R.; Malo, J.; Bath, J.; Andrew, J.; Turberfield, A. J. J. Am. Chem. Soc. 2004, 126, 16342-16343. (b) Rothemund, P. W. K.; Ekani-Nkodo, A.; Papadakis, N.; Kumar, A.; Fygenson, D. K.; Winfree, E. J. Am. Chem. Soc. 2004, 126, 1634416352.
Melting profiles of the four-helix weave tiles with and without the 10-base AT sticky-ends were also performed (Figure 3). These showed that the $T_{\mathrm{m}}$ of the individual tiles was $\sim 66{ }^{\circ} \mathrm{C}$ and that a weak melting transition just below $\sim 35{ }^{\circ} \mathrm{C}$ is observed for lattice dissolution. This weak lattice melting transition, together with the lack of distinct gel bands for lattices during PAGE analysis, could indicate that mica substrate acts to stabilize the formed arrays during AFM imaging, whereas they are relatively unstable and short-lived in solution.

For the eight-helix weave tile (8HT), lattice structures were observed both in gels and by AFM (Figure 4). The array had about the same size as for the 4HT but contained fewer individual tiles (due to the larger area of the 8HT), which is likely caused by increased flexibility of the longer tiles. Again, holes between the tiles were clearly visible, together with the 


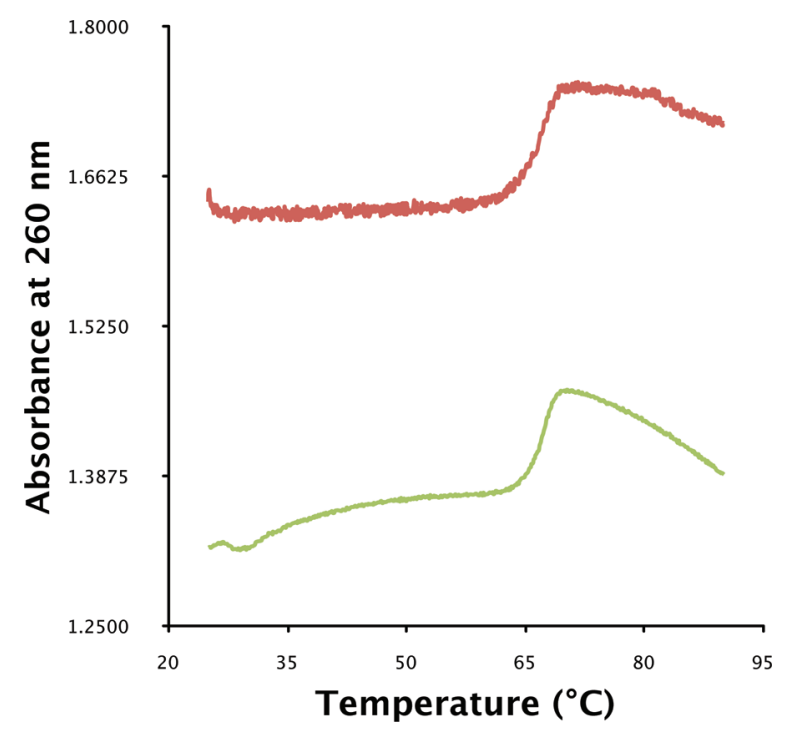

Figure 3. Melting profile of single four-helix weave tiles (red) and 4HT arrays with 10-base AT sticky-ends (green). $T_{\mathrm{m}}$ of the individual tiles is $\sim 66{ }^{\circ} \mathrm{C}$, and a weak, somewhat diffuse transition just below $35^{\circ} \mathrm{C}$ indicates a low $T_{\mathrm{m}}$ for the lattice structures.

expected prolonged length of the tile. No tube structures were ever observed in the 8HT array samples.

We then tested the use of 4HT as a scaffold for the nanoscale arrangement of biomolecules by replacing the sticky-ends with DNA aptamers that bind to thrombin, ${ }^{22,23}$ a serine protease that plays a key role in blood coagulation. The thrombin-binding aptamer (TBA) is a well-characterized aptamer that folds into a unimolecular guanine quadruplex and binds to thrombin's fibrinogen-recognition exosite with nanomolar affinity. ${ }^{24}$ By appending four aptamers upon the 4HT DNA weave scaffold, we hoped to increase the strength of the interaction between TBA and thrombin, and thereby prolong the blood coagulation time in a clotting assay. In the assay, commercially available platelin-activated blood plasma is recalcified, which triggers a cascade mechanism resulting in fibrin production and clot formation. The clotting time is measured in the coagulometer instrument by monitoring the movement of a metal bead placed in each well; the time recorded is when the bead is totally immobilized. By the addition of thrombin inhibiting factors, the clotting time is extended.

The aPTT assay was performed using a $0.25 \mu \mathrm{M}$ solution of aptamer tiles and comparing the clotting time with a $1 \mu \mathrm{M}$ solution of free aptamers giving the same total concentration

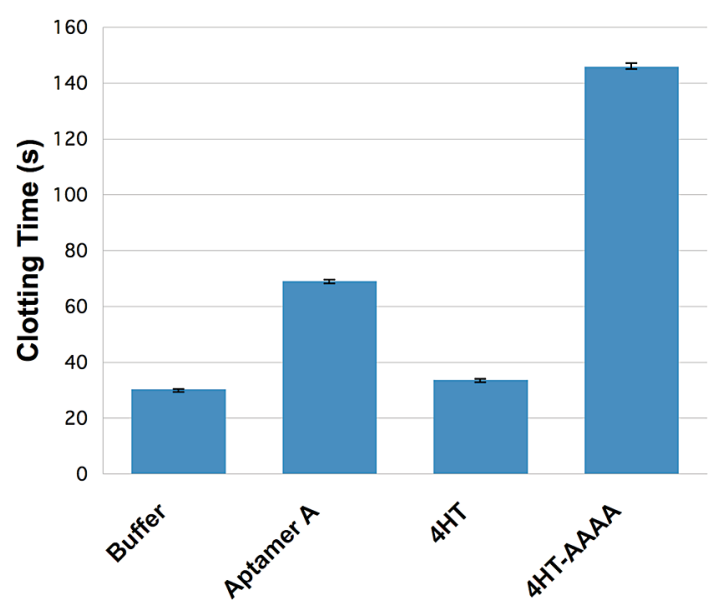

Figure 5. Coagulation assay results showing clotting times obtained using the activated partial thromboplastin time method. 4HT DNA weave tile without attached aptamer has activity similar to that negative control (buffer), while tile bearing four TBA aptamers (4HT-AAAA) shows the clotting time increased nearly 5-fold. The same concentration of free aptamer A gave only about a 2 -fold increase versus the buffer control. All reactions were performed in triplicate and averaged; error bars are shown.

of aptamer. As a negative control, simple 4HT tile without aptamers or sticky-ends was employed. The results (Figure 5) show that the $4 \mathrm{HT}$ weave tile displaying four copies of the aptamer prolonged the clotting time significantly compared to the buffer control, while the free aptamer provided only a modest increase. The negative control, 4HT weave tile with no aptamer, had no effect in the assay. The aptamer-bearing tiles are binding and inhibiting thrombin more than twice as well as free aptamer, probably due to a cooperative effect of having four TBA on each tile. The inherent flexibility of the weave tile design may also allow simultaneous associations by more than one aptamer without straining the overall molecular complex. Ongoing studies are investigating the co-attachment of another thrombin binding aptamer, ${ }^{25,17 \mathrm{~d}}$ which binds to the heparin binding exosite, to further strengthen the affinity for thrombin.

\section{Conclusion}

This study presented a new design for DNA tiles with the back and forth, woven strand routing also used in the origami architectures, but without Holliday junction-like crossovers to give a more flexible tile compared to those presented previously. One of the advantages of the weave tile design is its lower complexity compared to Holliday junction-based designs. In
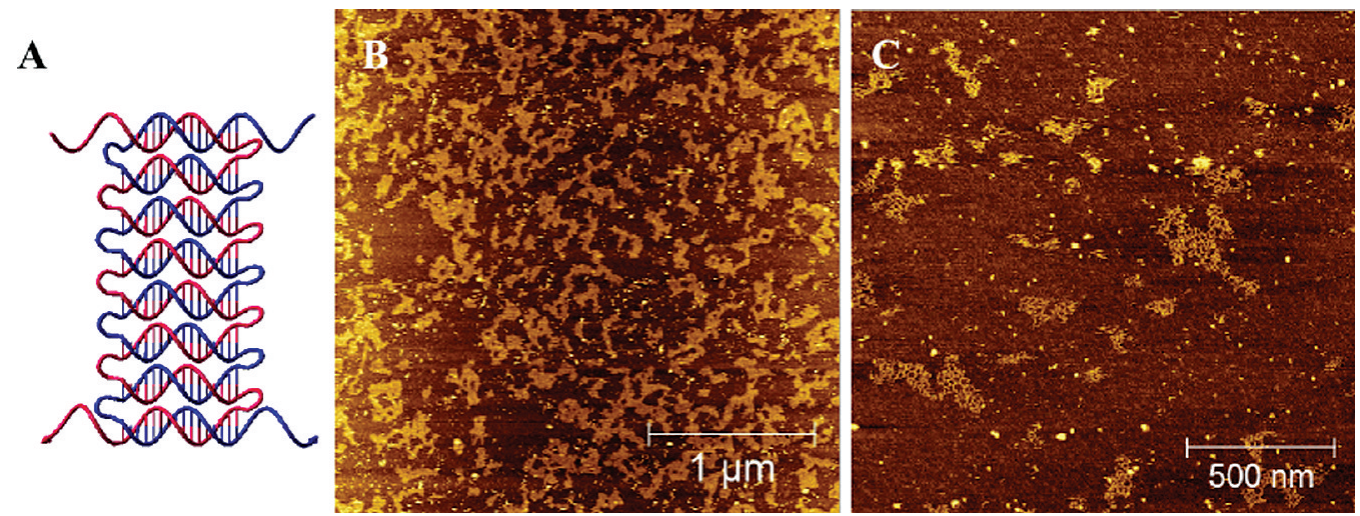

Figure 4. (A) Schematic drawing of eight-helix weave tile (8HT) showing the version with appended sticky-ends. (B,C) Typical AFM images of 8HT lattice. 
addition, since only two DNA strands are required per tile, assembly of oliogonucleotides into tiles is less susceptible to issues of incorrect strand stoichiometry that have been observed in other tile-based systems. Both the small 4HT and the larger $8 \mathrm{HT}$ were shown to form small patches up to $\sim 40000 \mathrm{~nm}^{2}$ of perfect lattice by the addition of short repeated AT sticky-end sequences. Applying GC sticky-ends only led to inferior lattice formation. Freshly annealed samples of 4HT also showed side products of tube-like structures connecting adjacent lattices; however, these were never observed for samples aged a few

(22) Bock, L. C.; Griffin, L. C.; Latham, J. A.; Vermaas, E. H.; Toole, J. J. Nature 1992, 355, 564-566.

(23) Padmanabhan, K.; Padmanabhan, K. P.; Ferrara, J. D.; Sadler, J. E.; Tulinsky, A. J. Biol. Chem. 1993, 268, 17651-17654.

(24) Macaya, R. F.; Schultze, P.; Smith, F. W.; Roe, J. A.; Feigon, J. Proc. Natl. Acad. Sci. U.S.A. 1993, 90, 3745. days at $4{ }^{\circ} \mathrm{C}$. When thrombin-binding aptamer was appended to the 4HT scaffold, it resulted in greater than a 2-fold increase in clotting time in coagulation assays as compared to free aptamer. Compared to uninhibited thrombin, the clotting time increase was 6-fold.

Acknowledgment. Financial support was provided by the U.S. National Science Foundation (CCF-08-29749, DMR-07-06397, CBET-08-35794, and OISE-06-24012) and by the Danish National Research Foundation. The authors thank Bruce Sullenger for coagulation assay expertise and access to equipment.

JA104456P

(25) Tasset, D. M.; Kubik, M. F.; Steiner, W. J. Mol. Biol. 1997, 272, 688-698. 